\title{
DESIGN CONSIDERATIONS FOR AN ISFET MULTIPLEXER AND AMPLIFIER
}

\section{PIET BERGVELD}

Department of Electrical Engineering, Twente University of Technology, $P O$ Box 217 , 7500 AE Enschede (The Netherlands)

(Recerved November 17, 1982, in revised form March 31, 1983, accepted May 6, 1983)

\begin{abstract}
Design considerations for the multiplexing of $\mathrm{pH}$-sensitive ISFETs are studied and discussed Experimental results with an ordinary multiplex circuit show transients of the order of several seconds if the output voltage of the applied amplifier is required within an accuracy of $05 \mathrm{mV}$ (corresponding to $001 \mathrm{pH}$ unit) These transients appear to result from thermal instability of the ISFETs during switching operations, and can be cancelled by guaranteeing a constant chip dissipation This necessitates the design of a special multiplexer circuit for multiplexing separate ISFETs The output voltage of the applied amplifier then appears to be stable within 1 mullsecond with the required accuracy
\end{abstract}

\section{Introduction}

After the introduction of the single ISFET sensor as a new electronic device for the measurement of chemical concentrations, several authors described devices specially designed to obtain selectivity for one particular Ion, such as $\mathrm{H}^{+}, \mathrm{Na}^{+}, \mathrm{K}^{+}$or $\mathrm{Ca}^{+}[1-5]$ In certain curcumstances, for example in biomedical applications, electrode arrays with a number of ISFETs for the simultaneous measurement of several ion concentrations would be very useful The basic ISFET concept is very suitable for this development because it makes use of standard microelectronic technology It is very easy to realize several FET structures in one chip and the various gate areas are well defined, membranes can easily be attached to each gate by means of photolithography, thus providing selectivity [6] In order to connect the different ISFETs to the 'outside world' it is usually troublesome to use separate leads for each source and drain, which means that the chip has to contain a multiplexer to connect one ISFET at a time to only two leads

A second application of ISFET multiplexing is for simultaneous testing of a number of ISFETs, usually of the same type, by means of one amplifier, 
connected to a parameter registration system In this case the multiplexer consists of a separate electronic switching circuit incorporated in the amplfier circuitry

This paper investigates the influence of connecting and disconnecting the ISFET source and drain on the electrical and chemical behaviour of the device In particular, the time response of the ISFET after switching will be examined, in relation to a given level of accuracy For example, it is useful to know the time required after switching to read out a pH-ISFET with 001 pH unit accuracy Furst, however, it is necessary to examine the practical conditions under which multiplexing is carried out

\section{Practical conditions of ISFET application}

In general, amplifier curcuits developed for the application of ISFETs are based on the source and drain follower concept, in which the drain current $I_{\mathrm{d}}$ is kept constant, but adjustable for calibration purposes, and where the applied drain-source voltage $V_{\text {ds }}$ is also kept constant [7] In the case of a multisensor application one such amplifier will be used, while the multiplexer connects the individual ISFETs in sequence to the amplifier A reference electrode determines the potential of the measuring solution with respect to the common terminal of the amplifier, usually the ground connection of the complete measuring and data processing system

Using only one ISFET and a corresponding amplifier, the drain current is usually set to a value that relates the output voltage of the amplifier to an appropriate calibration buffer For instance, for a pH ISFET this can mean that the drain current is adjusted to a value such that the amplifier output voltage $V_{\text {out }}=0 \mathrm{~V}$ corresponds to $\mathrm{pH}=7$ Using this procedure for a multiISFET sensor, it implies that the multiplexer should not only connect each separate individual ISFET to the amplifier, but should also switch the drain currents to the corresponding set-point values, which will differ between ISFETs due to production variations This problem is aggravated if the multi* sensor contains ISFETs which are selective for different ions

A more practical solution would be to apply the same drain current, as set by the amplifier, to all the separate sensors, and use a data processor to store the amplifier output voltages in relation to the ionic constituents of a calibration solution This means that the source and drain follower will adapt the source and drain voltage with respect to the common amplifier terminal (usually the grounded reference electrode), maintaining the same constant values of $I_{\mathrm{d}}$ and $V_{\mathrm{ds}}$ for each connected ISFET For practical reasons the sources of the different ISFETs of a multisensor are connected (one common diffusion in the chip) This means that the source and drain potentials of the ISFETs not connected vary with respect to the one which is momentarily connected

Electronically it is no problem to design an amplifier with a preconnected multiplexer, separate or integrated in the ISFET chip, which 
operates in the manner mentioned above It should, however, be investigated whether an ISFET will behave normally straight after being switched on, or if one should allow a certain time delay before sampling the amplifier output voltage

The data for transient ISFET phenomena already given in the literature [8] are not relevant here, because the measuring conditions were not directly applicable to a practical measurement set-up The given constants are defined as a $63 \%$ decay time, but the corresponding values of the amplifier output voltage cannot be extrapolated to the end-point values if the mechanism of the transient is unknown In this paper the output voltage of an amplifier with a pre-multiplexer, which sequentially switches two ISFETs, will be analysed and discussed

\section{The ISFETs and the ISFET amplifier}

The ISFETs used for the expermments were $\mathrm{Al}_{2} \mathrm{O}_{3}$ gate ISFETs with a sensitivity of approximately $50 \mathrm{mV}$ per decade $\left(\mathrm{H}^{+}\right)$The chips were mounted on a small glass fibre print plate with bond wires to conducting leads and isolated with epoxy, except for the gate area

The ISFET amplifier is a source and drain follower circuit (see Fig 1), whose operation has already been described in detall elsewhere [7] The source and drain connections are low-ohmic due to the feedback of the two supply operational amplifiers $A_{1}$ and $A_{2} \quad A_{3}$ is connected as a differential amplifier with unity gain $A_{4}$ is connected as an inverter. $A_{5}$ provides the open loop gain of the total system and $\mathrm{A}_{6}$ is a voltage follower The source and drain voltages were set a $500 \mathrm{mV}$, determined by the current source and $R_{1}$ The value of the drain current can be set by means of $V_{\text {ref }}$ and measured by means of $V_{\text {out }_{2}}$ where

$V_{\text {out }_{2}}=500 \mathrm{mV}+I_{\mathrm{d}}\left(R_{3}+R_{4}\right)$

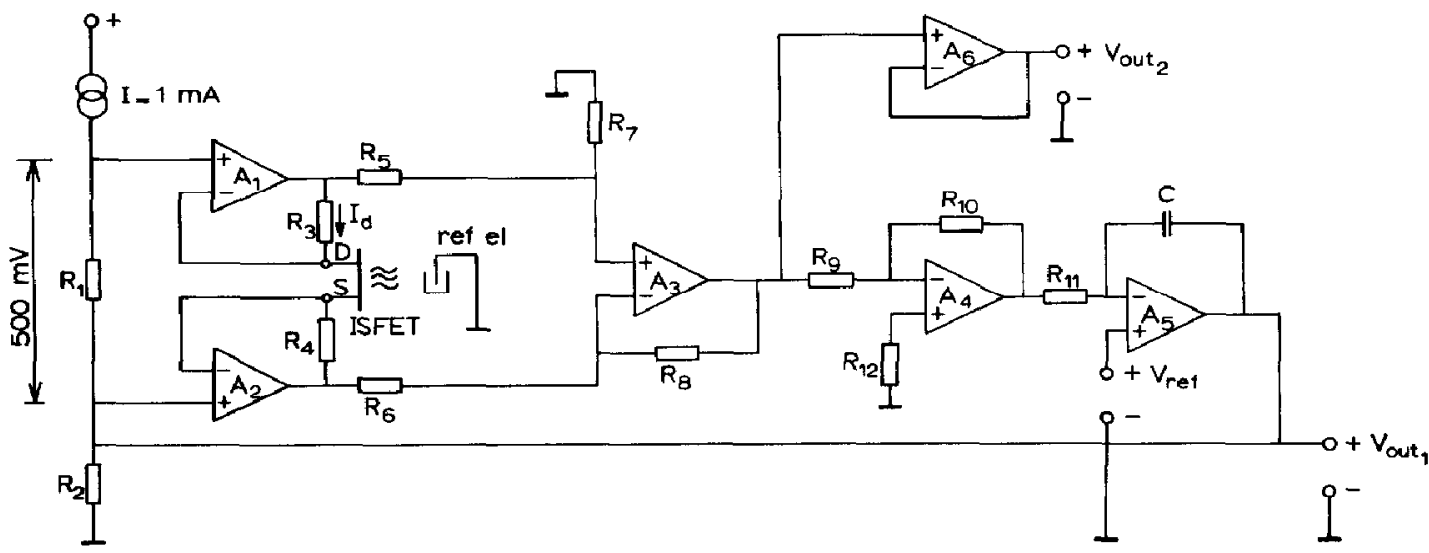

Fig 1 Amplifier circuit diagram according to the source and drain follower concept $R_{1}=R_{2}=500 \Omega, R_{3}=R_{4}=22 \mathrm{k} \Omega, R_{5}=R_{6}=R_{7}=R_{8}=R_{9}=R_{10}=R_{11}=R_{12}=33 \mathrm{k} \Omega$, $C=82 \mathrm{nF}, \mathrm{A}_{1}=\mathrm{A}_{2}=\mathrm{OP} 05, \mathrm{~A}_{3}=\mathrm{A}_{4}=\mathrm{A}_{5}=\mathrm{A}_{6}=\mathrm{MC} 1741$ 
$V_{\text {ref }}$ can be adjusted between 1 and $10 \mathrm{~V}$, thus controlling the drain current $I_{\mathrm{d}}$ between 11 and $215 \mu \mathrm{A} \quad V_{\text {out }}$ is equal to the source voltage with respect to ground and follows the ISFET surface potential withm an accuracy of 0999 This amplifier crrcuit operates very well in practice The bandwidth chosen is $0-5 \mathrm{kHz}$, determined by the value of the feedback capacitor $C$ of $\mathrm{A}_{5}$

\section{The multiplexer}

The multiplexer which has to be connected between the described amplifier and a set of ISFETs must either be integrated with the ISFET chip in the case of a multi-ISFET, or a separate curcuit for the simultaneous testing of single ISFETs For the purposes of this investigation, a simple design is chosen which is applied to two separate ISFETs and is not integrated to facilitate measurement of the actual operation at various points in the curcuit

Commercially avalable FET switches (Signetıcs SD 5001) are used, as shown in $F_{1}$ g 2 The bulk of the FET switches $F_{1}, F_{2}$ and $F_{3}$ is connected to the ground The connections $S$ and $D$ are applied to the source and drain inputs of the amplifier shown in Fig 1 The square-wave voltage generator can switch the drain $D_{1}$ of ISFET 1 or $D_{2}$ of ISFET 2 to $D$, while the sources and substrates of the two ISFETs are continuously connected to $\mathrm{S}$

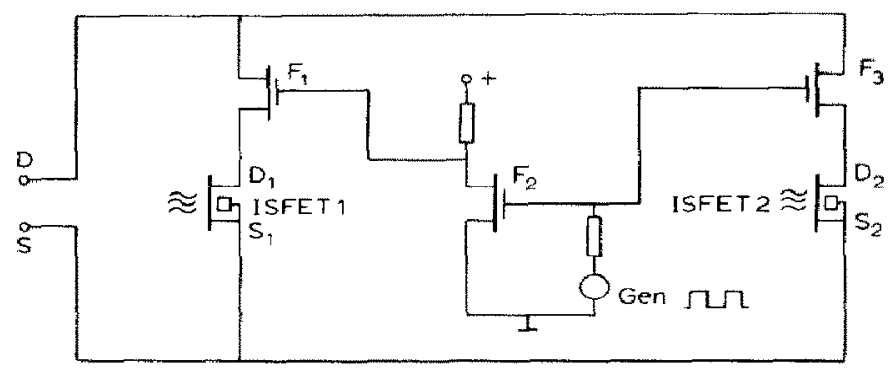

Fig 2 Circuit diagram for multiplexing two ISFETs applied to the amplifier circuit shown in Fig 1

\section{Results}

The tests were carried out in a buffer solution of $\mathrm{pH} 4$ Before testing the response of the ISFETs under drain current switching conditions, the response of the system was tested with only one ISFET constantly connected $\left(F_{1}\right.$ or $F_{3}$ on), while the reference electrode was connected to a square-wave voltage generator It appeared that the output voltage follows the input voltage within a millisecond, which is actually determined by the amplifier frequency response and is independent of the value of $I_{d}$, fixed by 
the reference voltage $V_{\text {ref }}$ This means that the ISFET, connected to the amplifier by means of the multiplexer, can handle a stepwise change within at least a millisecond During the actual multiplexing the reference electrode was connected to earth and long transients were observed Depending on the chosen value of the drain current, it took seconds before the output voltage of the amplifier was stable within the required accuracy of $05 \mathrm{mV}(001$ $\mathrm{pH})$

Figure 3 shows a typical result for the output voltage $V_{\text {out }_{1}}$ of the amplifier (see Fig 1 ) when $I_{d}$ was set to $20 \mu \mathrm{A}$ and ISFET 2 was connected with simultaneous disconnection of ISFET 1 The difference in $V_{\text {out }_{1}}$ for ISFET 1 and ISFET 2 results from differences in their characteristics Forcing the same current $I_{d}$ at the same drain-source voltage $V_{\mathrm{ds}}$ ensures that the source voltage (and the drain voltage) with respect to earth adapts itself to the characteristic of the ISFET that is switched on The differences may be of the order of volts, while the transients are of the order of millvolts The shape of the transients appeared to be a function of the drain current and changed in direction with increasing drain current

This transient phenomenon was also observed for a MOSFET with similar characteristics This suggests that electrochemical phenomena are not responsible for the ISFET transient Further, no real physical $R C$ time constant with such a high value can be found in the MOSFET, ISFET or multiplexer-amplifier system The drain current itself was checked to be constant ( $V_{\text {out }_{2}}$, Fig 1$)$ during multiplexing with no transients These observations and considerations mean that the transient phenomenon is the result of a shift in the $I_{\mathrm{d}}-V_{\mathrm{gs}}$ ISFET characteristic at the moment of switching on a particular ISFET Such a shift can be induced by an increase in temperature due to the dissipation of $I_{\mathrm{d}} V_{\mathrm{ds}}$ The shape and sign of the transient agree with these considerations, as shown in Fig 4, where recorded transients are shown at three points of the $I_{\mathrm{d}}-V_{\mathrm{gs}}$ characteristic, $\imath e$, below, beyond and at the isothermal point $\left(I_{\mathrm{d}}=80 \mu \mathrm{A}\right)$ The existence of the isothermal point for
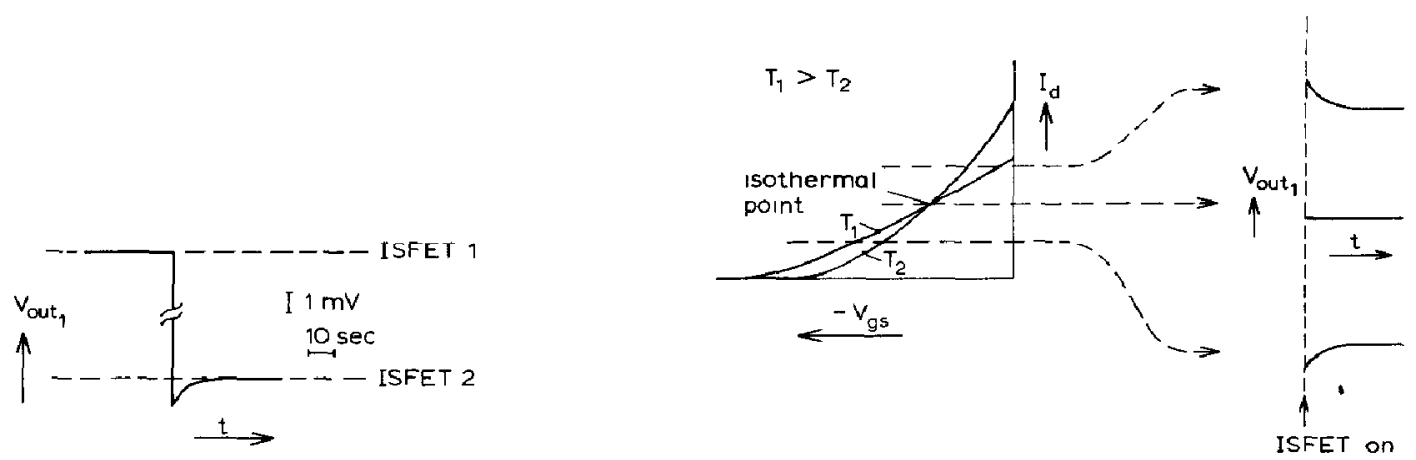

Fig 3 Typical recording of output voltage $V_{\text {out }}$ as a result of the multiplexing of two ISFETs

Fig 4 Typical recordings of the on-set of an ISFET at various values of the drain current $I_{\mathrm{d}}$ in relation to the $I_{\mathrm{d}}-V_{\mathrm{gs}}$ characteristic as a function of temperature 
ISFETs has been confirmed in the literature [9] Comparison of the heat conductivity values for water $\left(02 \mathrm{~mW} \mathrm{~cm}^{-1}{ }^{\circ} \mathrm{C}^{-1}\right)$ and $\mathrm{S}_{1}\left(2 \mathrm{~W} \mathrm{~cm}^{-1}{ }^{\circ} \mathrm{C}^{-1}\right)$ makes it clear that any heat dissipation in the silicon chip warms it up and possible cooling of the surrounding liquid has little effect It was observed that stirring does not influence the duration of the transient

As an example, we calculate the temperature effect for a chip $1 \mathrm{~mm}^{2} \times$ $200 \mu \mathrm{m}$, resulting from a realistic dissipation of $50 \mu \mathrm{W}\left(I_{\mathrm{d}}=100 \mu \mathrm{A}, V_{\mathrm{ds}}=\right.$ $500 \mathrm{mV}$ ) where thermal equilibrium is established after $25 \mathrm{~s}$ (the order of the observed time constant) Taking the silicon density as $232 \mathrm{~g} \mathrm{~cm}^{-3}$ gives a chip mass of $46 \times 10^{-4} \mathrm{~g}$, and the dissipation is $25 \times 50 \times 10^{-6} \mathrm{~J}$ Taking the specific heat of $\mathrm{S} 1$ as $076 \mathrm{~J} \mathrm{~g}^{-1}{ }^{\circ} \mathrm{C}^{-1}$, the temperature increase of the chip will thus be

$$
\Delta T=\frac{125 \times 10^{-4}}{076 \times 46 \times 10^{-4}}=035^{\circ} \mathrm{C}
$$

Knowing that the threshold voltage $V_{\mathrm{T}}$ of a MOSFET will shift by $\approx 2 \mathrm{mV}$ ${ }^{\circ} \mathrm{C}^{-1}[10]$, it is thus not surprising that transients of the order of millivolts will occur when an ISFET is switched on

\section{Conclusion and discussion}

The observations and calculation mentioned above suggest that during multiplexing, where ISFETs are switched on and off at drain current values below or beyond the isothermal operating point, the chip dissipation should be kept constant When the ISFETs are part of a multisensor with an integrated multiplexer, this constant dissipation requirement can be easily realized because one of the ISFETs is always in operation However, when separate individual ISFETs are multiplexed, for instance for test facilities, an alternative multiplexing system is required Figure 5 shows the principle of such a system, where as an example two ISFETs are connected in parallel most of the time After a certain time interval one of them is disconnected by the multiplexer for a very short time, for instance $10 \mathrm{~ms}$, while the second ISFET operates, determining the amplifier output voltage Thus means that both ISFETs are dissipating constantly but are read out sequentially However, the multiplexer circuit must connect and disconnect the measuring resistors of the amplifier $\left(R_{3}\right.$ and $\left.R_{4}\right)$ simultaneously, in order to adapt the amplifier to one ISFET or two ISFETs in parallel, and the two ISFETs should have approximately similar characteristics The time the ISFET is being measured $(10 \mathrm{~ms})$ is short with respect to possible temperature effects

Measurement results with a system as shown in Fig 5 indicate that the output voltage of the amplifier (F1g 1), where the source and drain measuring resistors $R_{3}$ and $R_{4}$ are replaced by the switched resistors $R_{3}, R_{3}{ }^{\prime}, R_{4}$ and $R_{4}{ }^{\prime}$ (Fig 5 ), gives a stable sampled value for each separate ISFET withın $1 \mathrm{~ms}$, fixed by the frequency response of the amplifier 


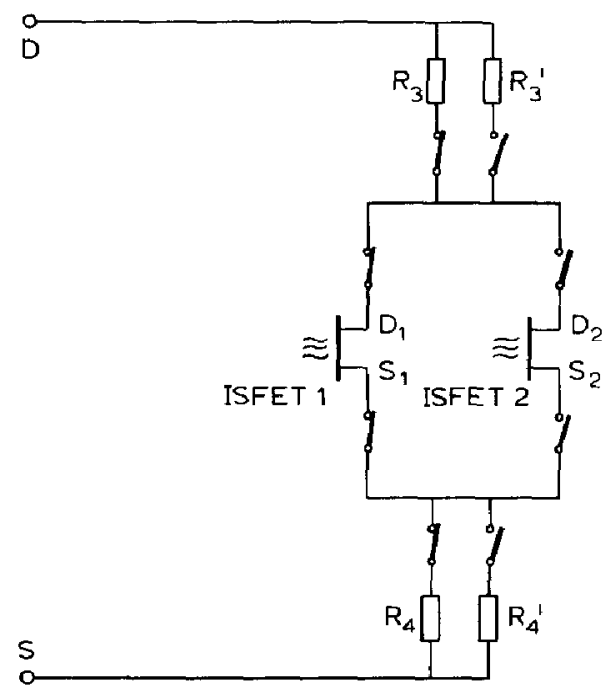

Fig 5 Principle of the alternative multiplexer switching system $R_{3}=R_{3}{ }^{\prime}=R_{4}=R_{4}{ }^{\prime}=$ $22 \mathrm{k} \Omega$

It will be clear that the system with two ISFETs can easily be extended to $n$ parallel ISFETs where $(n-1)$ are briefly disconnected sequentially to measure the remaining one

\section{Acknowledgement}

The author would like to thank L F M de Koning, B J F van Beynum and $M$ G Eggink for their help in designing and testıng the varıous amplifier and multiplexer crrcuits

\section{References}

1 K Shımada, M Yano, K Shıbatanı, Y Komoto, M Esashı and T Matsuo, Application of catheter tip ISFET for continuous in vivo measurement, Med Biol Eng Comp , $18(1980) 741-745$

$2 \mathrm{M}$ Esashı and $\mathrm{T}$ Matsuo, Integrated micro multı ion sensor using field effect of semlconductor IEEE Trans Blomed Eng, BME-25 (2) (March) (1978) 184 - 192

3 A Haemmerl, J Janata and $\mathbf{H}$ Brown, Ion-selective electrode for intracellular potassium measurements, Anal Chem , 52 (1980) $1179 \cdot 1182$

4 B A McKinley, J Saffle, W S Jordan, J Janata, S D Moss and D R Westenskow, In vivo contınuous monitoring of $\mathrm{K}^{+}$in animals using ISFET probes Med Instr, 14 (2) (March - April) (1980) 93 - 97

5 A U Ramsing, J Janata, J Ruzıcka and M Levy, Miniaturization in analytical chemistry - a combination of flow injection analysis and ion-sensitive field effect transistors for determination of $\mathrm{pH}$, and potassium and calcium ions, Analytica Chimica Acta, 118 (1980) $45 \cdot 52$

$6 \mathrm{G}$ Blackburn and $J$ Janata, The suspended mesh 10 selective field effect transistor, $J$ Electrochem Soc Solld State Sct Technol, 129 (11) (1982) 2580 - 2584 
$7 \mathrm{P}$ Bergveld, The operation of an ISFET as an electronic device, Sensors and $A c t u$ ators, 1 (1981) $17-29$

$8 \mathrm{~L} \quad \mathrm{R}$ Smith and $J$ Janata, Transient phenomena in ion sensitive field effect transistors, $J$ Electrochem Soc Solnd State Scl Technol, 127 (7) (July) (1980) 1599 1603

9 O Leistiko, The selectivity and temperature characteristics of ion sensitive field effect transistors, Physica Scripta, 18 (1980) 445450

$10 \mathrm{~K}$ Chow, $J$ P Rode, D H Seib and J D Blackwell, Hybrid infrared focal-plane arrays, IEEE Trans Electr Dev, ED 29 (1)(Jan )(1982) 313

\section{Biography}

Plet Bergweld was born in Oosterwolde, the Netherlands, on January 26, 1940 He received the M S degree in electrical engineering (electronics) from Eindhoven University of Technology, Eindhoven, the Netherlands, in 1965, and the $\mathrm{Ph} \mathrm{D}$ degree from Twente University of Technology, Enschede, the Netherlands, in 1973

Since 1965 he has been a member of the Bio-information Group, Department of Electrical Engineering, Twente University of Technology He is also a member of the Coordination Centre for Biomedical Engineering and a member of the Research Unit 'Sensors and Actuators' of this University In 1981 he became a member of the Semiconductor Group of the Foundation for Fundamental Research on Matter in the Netherlands

The subject of his dissertation was the ion-sensitive field-effect transistor (ISFET) and the OSFET $\mathrm{He}$ is involved in research on electronic measuring and stimulating methods in physiological systems, with special attention on in vivo biosensors He lectures on biomedical instrumentation to graduate students of Twente University of Technology and medical personnel of affiliated hospitals 